\section{MS29-P15 Large supramolecular assemblies of a bowl-shaped host}

Clive L. Oliver ${ }^{1}$

1. Department of Chemistry, University of Cape Town

email: clive.oliver@uct.ac.za

Nature is able to produce large, supramolecular assemblies of macromolecules with intricate complexities such as found in viruses and cellular membranes. ${ }^{1}$ These complicated structures are held together by non-covalent interactions which are ultimately crucial in the functioning of these complex biological systems. Small-molecule supramolecular chemists are inspired by these complex supramolecular systems in nature, however, large, synthetic, multi-component $(n>3)$ supramolecular assemblies which enclose chemical space are still relatively rare phenomena in the field of small-molecule, supramolecular chemistry. Atwood and MacGillivray reported the first example of such an assembly by showing that the bowl-shaped host molecule $C$-methylcalix[4]resorcinarene $\mathbf{1}$ can spontaneously assemble in a nitrobenzene solution to form a large, chiral, supramolecular assembly consisting of 6 molecules of $\mathbf{1}$ and 8 water molecules, the latter 'stitching' molecules of 1 into a hexameric assembly, $\mathbf{1}_{6}\left(\mathrm{H}_{2} \mathrm{O}\right)_{8}$ via $\mathrm{O}-\mathrm{H} . . \mathrm{O}$ hydrogen bonds. ${ }^{2}$ Despite the approximately 125 structures reported since this discovery containing 1 co-crystallised with various guest and/or solvent molecules, only one similar hexameric assembly of 1 was reported by Holman et al. where 6 of the 8 water molecules were replaced by 2 -ethylhexanol molecules. ${ }^{3}$ Hexameric assemblies of pyrogallolarenes and dodecameric assemblies of sulfonatocalix[4]arenes have also been reported. It is notable that in all these assemblies only one type of assembly per crystal structure was isolated in the solid-state. Here we present a crystallisation of $\mathbf{1}$ from 1-butanol, which yielded two different types of hexameric assemblies within the same crystal structure. Furthermore, the two unique assemblies are linked part of the time into a heterodimer of hexameric assemblies which we entitle a supra-heterodimer, a 38-component assembly consisting of 129 hydrogen bonds. To the best of our knowledge, the isolation of two different large, supramolecular assemblies $(\mathrm{n}>3)$ within the same crystal structure has not been observed before and neither has identical large supramolecular assemblies been shown to link into discrete units. In addition, we report a hexameric assembly of 1-propanol with 1 which increases the interior cavity size by simultaneous insertion of water and 1-propanol as the 'stitching' molecules, indicating a possible means of engineering the size of these cavities.

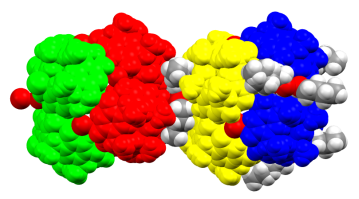

Figure 1. Space-filling representation of 38-component supra-heterodimer of 1

Keywords: large supramolecular assemblies, supra-heterodimer 